\title{
Model-Based Iterative Learning Control Applied to an Industrial Robot with Elasticity
}

\author{
W.B.J. Hakvoort, R.G.K.M. Aarts, J. van Dijk and J.B.Jonker
}

\begin{abstract}
In this paper model-based Iterative Learning Control (ILC) is applied to improve the tracking accuracy of an industrial robot with elasticity. The ILC algorithm iteratively updates the reference trajectory for the robot such that the predicted tracking error in the next iteration is minimised. The tracking error is predicted by a model of the closed-loop dynamics of the robot. The model includes the servo resonance frequency, the first resonance frequency caused by elasticity in the mechanism and the variation of both frequencies along the trajectory. Experimental results show that the tracking error of the robot can be reduced, even at frequencies beyond the first elastic resonance frequency.
\end{abstract}

\section{INTRODUCTION}

Several industrial applications put high demands on the speed and accuracy of robotic manipulators. For example, some laser welding applications require manipulation of the laser beam over a weld seam with an accuracy of $0.1 \mathrm{~mm}$ at speeds beyond $100 \mathrm{~mm} / \mathrm{s}$. This accuracy is often not reached by industrial six-axes robots with conventional controllers. Fortunately the repeatability of these robots is much better than their tracking accuracy and Iterative Learning Control (ILC) can be used to improve the tracking accuracy considerably.

ILC is a control technique to improve the tracking accuracy of systems that repetitively track the same reference over a finite time interval. The application of ILC to improve the accuracy of robotic manipulators has been studied extensively. Classically the focus is on improving the tracking accuracy of the robot's drives [1], [2], [3], [4], [5]. However, accurate motion of the drives does not necessarily imply accurate motion of a tool mounted to the robot [6]. This is partly caused by elasticity in the mechanism, which gives rise to resonance vibrations. The effect of elasticity on the robot dynamics has to be considered carefully in the design of an ILC algorithm to ensure convergence of the error at frequencies close to the elastic resonance frequencies. Investigations of the application of ILC to robotic manipulators with elasticity are scarce. A two-step ILC algorithm for a robot with elasticity in the transmission between the motor and the links was proposed in [7]. The proposed ILC algorithm improves the motion of the motor and the link side of the transmission in two alternating modes. The twostage approach adversely affects the convergence rate of the

W.B.J. Hakvoort is with the Netherlands Institute for Metals Research, Delft, The Netherlands

R.G.K.M. Aarts, J. van Dijk and J.B. Jonker are with the University of Twente, Enschede the Netherlands

Corresponding address: University of Twente, P.O. Box 217, $7500 \mathrm{AE}$ Enschede, The Netherlands, w.hakvoortenimr.nl tracking error. Alternative ILC algorithms for elastic robots with a higher rate of convergence were proposed in [8], [9]. Both algorithms employ a low-pass filter to filter out learning actions at high frequencies. Consequently the tracking error at high frequencies, e.g. the error caused by vibrations at the elastic resonance frequencies, is not compensated. The model-based ILC procedure proposed in [10] overcomes this disadvantage and is able to reduce the tracking error at frequencies close to the first elastic resonance frequency with a high rate of convergence. The model-based ILC procedure is applied successfully to a single link robot with linear timeinvariant dynamics. Extension of the procedure to six-axes industrial robots with multidimensional nonlinear dynamics is not trivial.

In this paper a model-based ILC procedure is proposed to reduce the tracking error of a six-axes industrial robot up to frequencies beyond the first elastic resonance frequency. The tracking error is not measured at the arm side of the transmission, as in most of the aforementioned publications, but at the tip of the robot where the tool is mounted. The tracking error of the tip is measured by a tip-mounted sensor as in [11]. A norm-optimal model-based ILC algorithm (see e.g. [10], [12], [13]) is used to compensate the measured error. The algorithm iteratively updates the reference trajectory for the robot to minimise the norm of the predicted tracking error in the next iteration. The implementation proposed in [13] is used to compute the iterative update of the reference trajectory efficiently. The tracking error in the next iteration is predicted by a model of the robot dynamics. The nonlinear robot dynamics is approximated by a locally linear model of which the parameters vary along the trajectory. The model includes the effect of elasticity to predict the tracking error at high frequencies. The variation of the servo resonance frequency and the first elastic resonance frequency along the trajectory is modelled by the variation of the model parameters. The model parameters are estimated from measurements of the robot's dynamic response with system identification techniques [14]. The experimental results in this work show that the tracking error of the robot tip can be compensated up to frequencies beyond the first elastic frequency, resulting in a substantial decrease of the error.

\section{ITERATIVE LEARNING CONTROL}

In this work a norm-optimal ILC algorithm is used. The implementation was previously described in [13]. This section describes the algorithm and its convergence properties. 


\section{A. ILC algorithm}

The dynamics of the robot is modelled as linear timevariant. Linear time-variant dynamics can be described by the following state space system

$$
\begin{aligned}
x_{i+1} & =A_{i} x_{i}+B_{i} u_{i}, \\
y_{i} & =C_{i} x_{i}+w_{i},
\end{aligned}
$$

where $u_{i}$ is the input that is modified by the ILC algorithm, $y_{i}$ is the output that is controlled by the ILC algorithm, $x_{i}$ the state vector and $w_{i}$ the output disturbance. Subscript $i$ denotes the time index and $N_{i}$ is the total number of times steps.

Analyses of the iterative behaviour of discrete systems is facilitated by the matrix description of system dynamics [4], [5], [12]. In the matrix description all time instances of a discrete time signal are concatenated into a single vector. The system dynamics is represented by a matrix that maps the vector of inputs to the vector of outputs. Rewriting (1) in the matrix description gives

$$
\mathbf{y}=\mathbf{G u}+\mathbf{y}_{0}+\mathbf{w},
$$

where $\mathbf{y}=\left[\begin{array}{llll}y_{1}^{T} & y_{2}^{T} & \ldots & y_{N_{i}}^{T}\end{array}\right]^{T}, \mathbf{u}=\left[\begin{array}{llll}u_{1}^{T} & u_{2}^{T} & \ldots & u_{N_{i}}^{T}\end{array}\right]^{T}$, etcetera. The system matrix $\mathbf{G}$ and the effect of the initial states on the output $\mathbf{y}_{0}$ are given by

$$
\begin{aligned}
\mathbf{G} & =\left[\begin{array}{cccc}
0 & 0 & \cdots & 0 \\
C_{2} B_{1} & 0 & \ddots & 0 \\
C_{3} A_{2} B_{1} & C_{3} B_{2} & \ddots & 0 \\
C_{4} A_{3} A_{2} B_{1} & C_{4} A_{3} B_{2} & \ddots & 0 \\
\vdots & \ddots & \ddots & \vdots \\
C_{N_{i}} \prod_{i=N_{i}-1}^{2} A_{i} B_{1} & C_{N_{i}} \prod_{i=N_{i}-1}^{3} A_{i} B_{2} & \ldots & C_{N_{i}} B_{N_{i}-1}
\end{array}\right], \\
\mathbf{y}_{0} & =\left[\begin{array}{c}
C_{1} A_{0} \\
C_{2} A_{1} A_{0} \\
C_{3} A_{2} A_{1} A_{0} \\
\vdots \\
C_{N} \prod_{i=N_{i}-1} A_{i}
\end{array}\right] x_{0} .
\end{aligned}
$$

Iterative Learning Control should reduce the tracking error e, which is defined as

$$
\mathbf{e}=\mathbf{r}-\mathbf{y}=\mathbf{r}-\mathbf{G u}-\mathbf{w}-\mathbf{y}_{0},
$$

where $\mathbf{r}$ is the reference for the output $\mathbf{y}$. The reference is identical in each iteration. Furthermore it is assumed that the effect of the initial states $\mathbf{y}_{0}$ and the disturbance $\mathbf{w}$ are identical in each iteration. With these assumptions system (5) can be rewritten as

$$
\mathbf{e}^{k}=\mathbf{d}-\mathbf{G u}^{k}
$$

where the superscript $k$ denotes the iteration number and

$$
\mathbf{d}=\mathbf{r}-\mathbf{w}-\mathbf{y}_{0},
$$

contains all signals that do not depend on the input and are assumed to be iteration invariant.

The norm-optimal ILC algorithm iteratively updates the input $\mathbf{u}^{k+1}$ such that the estimated error in the next iteration $\hat{\mathbf{e}}^{k+1}$ is minimised while the growth of the input is limited, thus

$$
\mathbf{u}^{k+1}=\underset{\mathbf{u}^{k+1}}{\arg \min }\left(\hat{\mathbf{e}}^{k+1 T} \mathbf{V} \hat{\mathbf{e}}^{k+1}+\Delta \mathbf{u}^{k+1 T} \mathbf{W} \Delta \mathbf{u}^{k+1}\right),
$$

where $\Delta \mathbf{u}^{k+1}=\mathbf{u}^{k+1}-\mathbf{u}^{k}$ and $\mathbf{V}$ and $\mathbf{W}$ are weighing matrices. Matrices $\mathbf{V}$ and $\mathbf{W}$ are block-diagonal matrices with the time-dependent positive definite symmetric matrices $V_{i}$ and $W_{i}$ on their respective diagonals. The error estimate $\hat{\mathbf{e}}^{k+1}$ is obtained from a model with time-varying state-space matrices $\hat{A}_{i}, \hat{B}_{i}, \hat{C}_{i}$. Analogously to (3) an estimated system matrix $\hat{\mathbf{G}}$ can be constructed from these state space matrices. The error estimate $\hat{\mathbf{e}}^{k}$ is obtained by replacing system matrix $\mathbf{G}$ in (6) by its estimate $\hat{\mathbf{G}}$. Subtracting the error estimate for trial $k$ from the estimate for trial $k+1$, gives the following expression

$$
\hat{\mathbf{e}}^{k+1}=\mathbf{e}^{k}-\hat{\mathbf{G}}\left(\mathbf{u}^{k+1}-\mathbf{u}^{k}\right) .
$$

Substituting this expression in (8) and minimising with respect to $\mathbf{u}^{k+1}$ gives the following input update equation

$$
\mathbf{u}^{k+1}=\mathbf{u}^{k}+\mathbf{L} \mathbf{e}^{k},
$$

where the learning matrix $\mathbf{L}$ is given by

$$
\mathbf{L}=\left(\hat{\mathbf{G}}^{T} \mathbf{V} \hat{\mathbf{G}}+\mathbf{W}\right)^{-1} \hat{\mathbf{G}}^{T} \mathbf{V} .
$$

Updating the input according to (10) with the learning matrix in (11) reduces the error optimally in the sense of (8). Equation (10) is used iteratively; First the tracking error is measured for some initial input. Thereafter each iteration consists of updating the input according to (10) and measuring the tracking error for the new input. An algorithm to compute the input update in (10) efficiently, without the need to construct the (large) learning matrix, is proposed in [13].

\section{B. Convergence}

The input of the system in (6) is computed iteratively according to (10). The sequence of inputs converges monotonically if

$$
\bar{\sigma}(\mathbf{I}-\mathbf{L G}) \leq 1,
$$

where $\bar{\sigma}(\cdot)$ denotes the largest singular value. Condition (12) holds for the learning matrix in (11) if $\hat{\mathbf{G}}=\mathbf{G}$, i.e. if the system is modelled correctly. However, in practice, model $\hat{\mathbf{G}}$ does not model the dynamics $\mathbf{G}$ exactly. The sequence of inputs diverges if the input update that reduces the error predicted by model $\hat{\mathbf{G}}$ changes the error of the real system $\mathbf{G}$ such that the norm of the input update in the next iteration increases. A common way to solve this problem is to filter out all inputs to which the response of the system is not modelled with sufficient accuracy. The update in (10) is changed to

$$
\mathbf{u}^{k+1}=\mathbf{Q}\left(\mathbf{u}^{k}+\mathbf{L} \mathbf{e}^{k}\right),
$$


where $\mathbf{Q}$ is the robustness filter. For this update equation the sequence of inputs converges monotonically if

$$
\bar{\sigma}(\mathbf{Q}(\mathbf{I}-\mathbf{L G})) \leq 1 .
$$

The robustness filter thus increases the robustness for modelling errors. Provided that the sequence of inputs converges, the sequence of errors converges to

$$
\mathbf{e}^{\infty}=\left(\mathbf{I}-\mathbf{G}(\mathbf{I}-\mathbf{Q}+\mathbf{Q} \mathbf{L G})^{-1} \mathbf{Q} \mathbf{L}\right) \mathbf{d} .
$$

If $\mathbf{Q}=\mathbf{I}$ (no filter) and if $\mathbf{L}$ and $\mathbf{G}$ are invertible then $\mathbf{e}^{\infty}=0$. On the other side, if the robustness filter is not unity then the final error is nonzero. Trivially, $\mathbf{e}^{\infty}=\mathbf{d}$ if $\mathbf{Q}=0$ (no learning). Thus although the robustness-filter increases the robustness for modelling errors it also results in a nonzero final error.

For practical applications of ILC to mechanical systems the robustness filter $\mathbf{Q}$ is commonly implemented as a low-pass filter. The high-frequency part of the input needs to be filtered out if the effect of elasticity on the highfrequency dynamics is not modelled with sufficient accuracy. The necessity of the low-pass robustness filter is illustrated by the following example. Suppose the ILC algorithm is applied to improve the tracking of a single robot link with a collocated PD feedback controller. The reference for the motor position is iteratively updated by the ILC algorithm to reduce the tracking error that is measured at the link side of the transmission. The transmission between the motor and the link is elastic, but this elasticity is neglected in the model. The difference in the phase of the frequency response of the model and the real system is more than $90 \mathrm{deg}$ beyond the resonance frequency. The input update that reduces the predicted error beyond this frequency thus increases the error of real system. The growth of the error increases the input update in the next iteration and thus the sequence of inputs diverges. A low-pass robustness filter is needed to filter out the input updates at frequencies beyond the elastic resonance frequency. Disadvantageously the highfrequency components of the error are not compensated and a non-zero error remains. Thus compensation of the tracking error at high frequencies requires adequate modelling of the elasticity in the mechanism.

\section{MODELLING}

Application of the ILC algorithm in the previous section to an industrial robot requires a model of the robot to predict its tracking error. Compensation of the tracking error at high frequencies requires a sufficiently accurate model of the high-frequency dynamics. The high-frequency dynamics is affected by the elasticity in the robot mechanism. This section presents a model structure that is suited to model the high-frequency dynamics of the robot and a procedure to identify the parameters of the model.

\section{A. Model structure}

It is assumed that a tip-mounted sensor directly measures the relevant subset of linear and angular components of the tracking error of a tool mounted to the robot. These measurements are expressed in a local coordinate system of the sensor. The measured tracking error is reduced with ILC by changing the motion reference for the robot in the directions measured by the sensor. If the robot would perfectly track the motion reference, then the relation between the motion reference and the measurements would be unity. In reality the relation is not unity because of the dynamics of the robot mechanism and the controller.

In many industrial systems the dynamics of the controller are linear time-invariant, while the dynamics of the robot mechanism are non-linear. The non-linear closed-loop dynamics can be linearised for small motions and the linearised dynamics depend on the configuration of the robot. In this work the varying dynamics are modelled by interpolating linear models along the trajectory of the robot. The relation between the correction of the motion reference $u_{i}$ and the measured tracking error $e_{i}$ is modelled as

$$
e_{i}=\sum_{m=1}^{N_{m}} p_{m, i}\left(\sum_{\tau=0}^{N_{b}} \tilde{B}_{m, \tau} u_{i-\tau-N_{k}}-\sum_{\tau=1}^{N_{a}} \tilde{A}_{m, \tau} e_{i-\tau}\right)+e^{0}{ }_{i},
$$

where $e^{0}{ }_{i}$ is the tracking error for $u_{i}=0$. The parameters $p_{m, i}$ interpolate the elements of matrices $\tilde{A}_{m, \tau}$ and $\tilde{B}_{m, \tau}$ of the $N_{m}$ linear models along the trajectory. The interpolation parameters $p_{m, i}$ are defined as

$$
p_{m, i}=\left\{\begin{array}{lll}
m-\frac{d_{i}}{D} & \text { if } & \frac{d_{i}}{D} \leq m<\frac{d_{i}}{D}+1, \\
2-m+\frac{d_{i}}{D} & \text { if } & \frac{d_{i}}{D}+1 \leq m<\frac{d_{i}}{D}+2, \\
0 & \text { else, }
\end{array}\right.
$$

where $d_{i}$ is the distance along the trajectory at time $i$ and $D$ is the total distance along the trajectory divided by the number of models minus one, i.e. $D=d_{N_{i}} /\left(N_{m}-1\right)$. The elements of matrices $\tilde{A}_{m, \tau}$ and $\tilde{B}_{m, \tau}$ are estimated by the procedure described in the next subsection.

\section{B. Identification procedure}

The elements of matrices $\tilde{A}_{m, \tau}$ and $\tilde{B}_{m, \tau}$ of the robot model are estimated from measurements of the robot's dynamic response using system identification techniques [14].

The dynamic response is measured in two steps. First the robot is moved along the trajectory to measure $e^{0}{ }_{i}$. Secondly the tracking error $e_{i}$ is measured along the trajectory for a set of small excitations of the motion reference $u_{i}$.

The elements of matrices $\tilde{A}_{m, \tau}$ and $\tilde{B}_{m, \tau}$ are estimated by minimising the quadratic norm of the difference between the left and the right hand side of (16) for all time steps and all sets of measurements. Low-pass filtering of both sides of (16) can be used to improve the fit at low frequencies. The parameter estimate can be computed efficiently since the right hand side of (16) is linear in the unknown parameters $\tilde{A}_{m, \tau}$ and $\tilde{B}_{m, \tau}$. Note that the proposed identification procedure does not minimise the error in the estimate of $e_{i}$ since $e_{i}$ appears on both sides of (16). Similarly to the identification of ARX models in [14] this might result in a biased estimation of the parameters. Nevertheless the proposed identification procedure is preferred because of its efficient computation. 


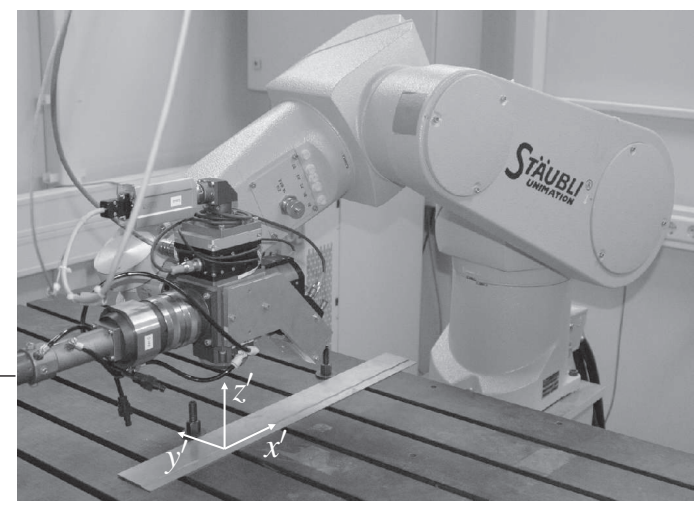

Fig. 1. The Stäubli RX90 robot carrying a welding head with integrated seam tracking sensor

\section{EXPERIMENTAL RESULTS}

This section presents the results of the application of the proposed ILC procedure to an industrial robot with a tip-mounted sensor. The experimental setup is described in subsection IV-A. Subsection IV-B treats the identification of the model of the robot dynamics. In subsection IV-C the results of the application of the ILC procedure are presented.

\section{A. Experimental setup}

The experimental setup, depicted in Fig. 1, consists of a six-axes industrial Stäubli RX90 robot that carries a welding head with integrated seam-tracking sensor. The sensor measures the position of a weld seam relative to the welding head. The weld seam consists of two overlapping metal strips. The upper metal strip has a serrated profile with a period of $50 \mathrm{~mm}$ and an amplitude of $2 \mathrm{~mm}$. The location of the seam is known approximately and ILC is used to improve the tracking of the seam. The seam-tracking sensor measures the tracking error with respect to the same weld seam repetitively. The location of the seam is not changed during the iterations. The seam is welded after the tracking error is reduced sufficiently.

The repeatability of the Stäubli RX90 robot is $\pm 0.02 \mathrm{~mm}$. The motion of its drives is controlled by the industrial CS8 controller. The motion of each drive is controlled independently with a cascaded velocity and position feedback loop. Velocity and acceleration feedforwards are used to improve the tracking behaviour. Conventionally position and velocity setpoints for the drive controllers are computed by the trajectory generator of the CS8 controller. For this work the Real Time Robot Controller Abstraction Layer (RTRCAL) [15] is used to supply the setpoints to the drive controllers directly at a sample rate of $250 \mathrm{~Hz}$. The ILC algorithm updates the reference for the robot motion in the local coordinate system of the sensor. This update is multiplied with the inverse of the local kinematic Jacobian matrix [16] to obtain the update of the position setpoints for the robot drives. The Jacobian matrix relates small changes of the drive position to small changes of sensor position in the local coordinate system of the sensor. The velocity setpoints are computed by numerical differentiation.

The position of the weld seam with respect to the welding head is measured by the industrial Falldorf seam tracking sensor, which is based on optical triangulation. The sensor measures the location of the seam in its local $x^{\prime} y^{\prime} z^{\prime}$ coordinate system with the origin located at the focus point of the welding laser and the axes oriented as illustrated in Fig. 1. The seam-tracking sensor only measures the position of the seam in the $y^{\prime}$ and $z^{\prime}$-direction. Since the origin of the sensor's coordinate system is the focus point of the welding laser, the tracking error is equal to the distance of the seam measured by the sensor. A synchronisation protocol has been implemented to measure the tracking error synchronously with the robot motion [17].

Initially the robot is commanded to move along a straight line in the $x^{\prime}$-direction from the initial position at $800 \mathrm{~mm}$ from the robot base towards the final position at $400 \mathrm{~mm}$ from the base. The initial robot configuration is depicted in Fig. 1. The velocity profile is trapezoidal with maximum velocity of $400 \mathrm{~mm} / \mathrm{s}$ and a maximum acceleration of $1600 \mathrm{~mm} / \mathrm{s}^{2}$. The tracking error in the $y^{\prime}$ and $z^{\prime}$-direction for the initial motion reference is shown in Fig. 2. The serrated profile of the metal strip is clearly visible. A misalignment of the metal strip results in an offset and a linear trend in both directions. ILC is used to update the motion reference in the $y^{\prime}$ and $z^{\prime}$-direction iteratively, such that the metal strip is traced accurately in those directions. The $x^{\prime}$-position and the angle of the seam are not measured and the motion reference is not updated in these directions.

The ILC algorithm, which is described in section II-A, requires a model of the robot dynamics to predict the tracking error. The ILC algorithm can only compensate the error in the frequency range in which the robot dynamics is modelled with sufficient accuracy. The frequency content of the error thus gives the frequency range in which the robot should be modelled. Fig. 3 shows the maximum absolute value of the initial error in Fig. 2 that remains after filtering with a high-pass filter as a function of the cut-off frequency of the high-pass filter. The figure shows that the maximum absolute tracking error is smaller than $0.1 \mathrm{~mm}$ for a cutoff frequency of at least $21 \mathrm{~Hz}$. Part of the tracking error is caused by non-repetitive effects like sensor noise and the limited repeatability of the robot mechanism. These nonrepetitive effects cannot be compensated by ILC and thus the final error will be slightly larger than the error indicated in Fig. 3. Therefore the motion reference has to be compensated up to a frequency beyond $21 \mathrm{~Hz}$ to reduce the tracking error to $0.1 \mathrm{~mm}$.

\section{B. Identification results}

The ILC algorithm requires a model of the relation between a change of the motion reference in the $y^{\prime}$ and $z^{\prime}$ direction and the resulting change of the measurements of the seam tracking sensor. This relation is modelled with the model structure described in subsection III-A and the identification procedure described in subsection III-B. 


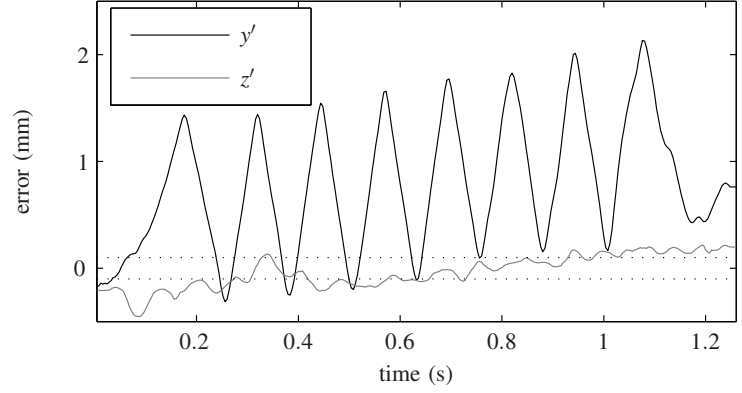

Fig. 2. Initial tracking error

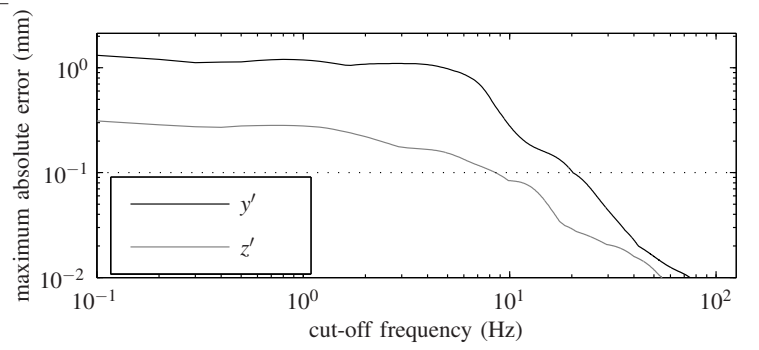

Fig. 3. Maximum absolute value of the initial tracking error after filtering with a high-pass filter

First the tracking error is measured along the trajectory for the initial motion reference to obtain $e^{0}{ }_{i}$. Thereafter the tracking error is measured along the trajectory while a multisine excitation is added to the motion reference. The response to the excitation of the motion reference in the $y^{\prime}$ and $z^{\prime}$ is measured separately. The measurements are repeated for four different multisine realisations. Each multisine excitation is composed of sine wave components with frequencies up to $40 \mathrm{~Hz}$. The phase of each component is chosen randomly. The amplitude of the components is equal up to $10 \mathrm{~Hz}$ and beyond $10 \mathrm{~Hz}$ the relative amplitude of the components decreases linearly with the frequency. The maximum amplitude of the multisine is scaled to $0.5 \mathrm{~mm}$. The robot's response can be measured and modelled up to $40 \mathrm{~Hz}$ with the selected multisine excitation. According to the previous subsection the tracking error can be reduced to $0.1 \mathrm{~mm}$ if the robot dynamics is modelled accurately up to about $21 \mathrm{~Hz}$. A larger frequency band is taken to investigate if the tracking error can be reduced further.

Three linear models are interpolated along the trajectory to model the variation of the dynamics along the trajectory, i.e. $N_{m}=3$. The delay between the commanded motion and the measured response is two samples, so $N_{k}=2$. The selection of $N_{a}$ and $N_{b}$ is based on the comparison of the response of the model and the real robot. The response is compared for a set measurement data that is not used for the identification of the parameters. The measurements and the response of the model match sufficiently accurate for $N_{a}=5$ and $N_{b}=3$.

Fig. 4 shows the local frequency response of the identified model at the start-, mid- and endpoint of the trajectory. Note that at low frequencies the robot tracks the reference almost perfectly. Between $10-20 \mathrm{~Hz}$ the servo resonance frequency
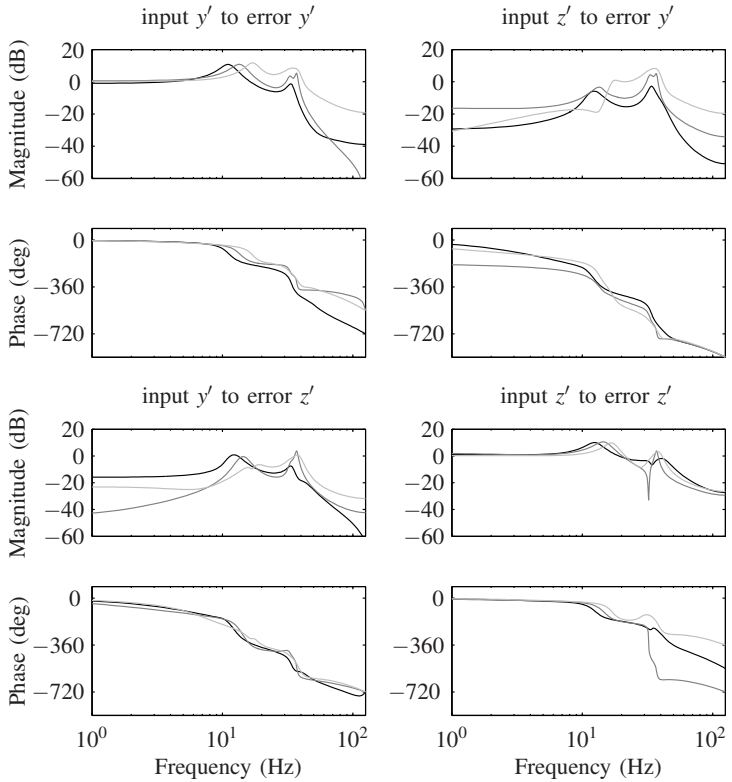

Fig. 4. Local frequency response of the identified model black: startpoint, dark grey: midpoint, light grey: endpoint

of the closed-loop system is visible. The servo resonance frequency depends on the position along the trajectory, because the inertia of the mechanism acting on the drives depends on the robot configuration. Slightly above $30 \mathrm{~Hz}$ a peak due to the first elastic resonance frequencies is visible. The height of the peak clearly depends on the robot configuration, but the frequency depends only slightly on the configuration. Since the multisine excitation, which was used to obtain the data for identification, contains frequencies up to $40 \mathrm{~Hz}$, the model is not reliable at frequencies beyond $40 \mathrm{~Hz}$.

The model with the estimated parameters is converted to its time-varying state-space representation for use with the proposed ILC algorithm. The next section describes the results of the application of ILC.

\section{Reduction of the tracking error}

The norm-optimal ILC algorithm described in subsection II-A is employed to reduce the tracking error of the Stäubli RX90 robot, measured with the seam tracking sensor. The weighing matrices are selected as

$$
V_{i}=I, \quad W_{i}=0.5 I
$$

where $I$ is the identity matrix. The robustness-filter is implemented as a zero-phase low-pass filter. Three different values for the cut-off frequency of the low-pass filter are tested; $20 \mathrm{~Hz}, 30 \mathrm{~Hz}, 40 \mathrm{~Hz}$. From Fig. 3 it is expected that the tracking error is not reduced below $0.1 \mathrm{~mm}$ for a cut-off frequency of $20 \mathrm{~Hz}$.

The ILC algorithm is run for 20 iterations. Fig. 5 shows the maximum absolute tracking error in each of the iterations. The figure shows that ILC is able to reduce the tracking error substantially. The higher the cut-off frequency the smaller the maximum absolute tracking error that remains 

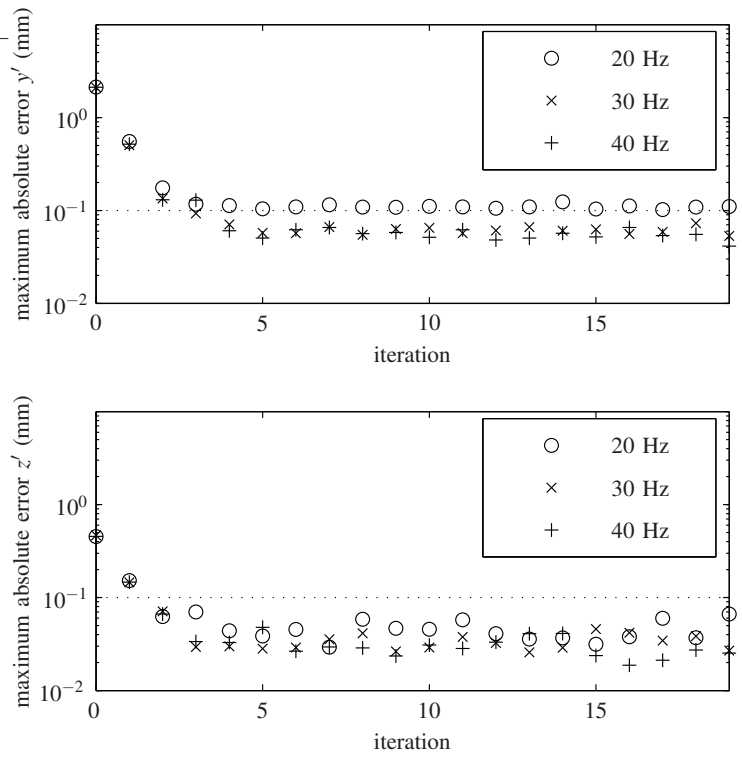

Fig. 5. Maximum absolute tracking error in each of the iterations for different values of the cut-off frequency of the robustness filter.

after convergence. The maximum absolute tracking accuracy is larger than $0.1 \mathrm{~mm}$ for a cut-off frequency of $20 \mathrm{~Hz}$, which was expected from Fig. 3. The tracking error is reduced below $0.1 \mathrm{~mm}$ for a cut-off frequency of $30 \mathrm{~Hz}$ and $40 \mathrm{~Hz}$. The remaining maximum absolute tracking error is larger than indicated in Fig. 3, which is probably caused by non-repetitive effects like the repeatability of the robot mechanism and inaccuracies of the sensor measurement. Increasing the cut-off frequency beyond $40 \mathrm{~Hz}$ will improve the tracking only slightly because non-repetitive effects are the main cause of the remaining error.

Fig. 5 shows that the tracking error converges even if the cut-off frequency of the robustness filter is $40 \mathrm{~Hz}$. Fig. 4 shows that the first elastic resonance frequency of the modelled system is below $40 \mathrm{~Hz}$ and that the phase of the (local) frequency response changes $180 \mathrm{deg}$ at that frequency. As discussed in subsection II-B the difference in phase of the frequency response of the model and the real system should be less than 90 deg to avoid divergence of the sequence of inputs. The reduction of the tracking error with inputs beyond the first resonance frequency is possible because the location of the resonance frequency is modelled sufficiently accurate.

\section{CONCLUSIONS}

In this paper a procedure is proposed to improve the tracking of a tool mounted to an industrial robot with elasticity. The tracking error of the tool is measured directly and a norm-optimal ILC algorithm iteratively updates the reference motion for the robot to reduce the predicted tracking error in the next iteration. The tracking error is predicted with a model of the robot dynamics. The robot dynamics vary along the trajectory, which is modelled by interpolating the parameters of a linear model along the trajectory.

Experimental results show that the tracking error of a laser welding head mounted to an industrial robot can be reduced substantially using the proposed procedure. The tracking error is even reduced at frequencies beyond the first elastic resonance frequency. The obtained accuracy is suited for demanding laser welding applications. The remaining error is mainly caused by non-repetitive effects, which cannot be compensated with ILC.

\section{ACKNOWLEDGMENTS}

This research was carried out under project number MC8.03161 in the framework of the Strategic Research programme of the Netherlands Institute for Metals Research in the Netherlands (www.nimr.nl)

\section{REFERENCES}

[1] S. Arimoto, S. Kawamura, and F. Miyazaki, "Bettering operation of dynamic systems by learning: A new control theory for servomechanism or mechtatronic systems." Journal of Robotic Systems, vol. 1, no. 2, pp. 123-140, March 1984.

[2] S. Kawamura, F. Miyazaki, and S. Arimoto, "Realization of robot motion based on a learning method," IEEE Transactions on Systems, Man and Cybernetics, vol. 18, no. 1, pp. 126-134, January/February 1988.

[3] T. Kavli, "Frequency domain synthesis of trajectory learning controllers for robot manipulators," Modeling, identification and control, vol. 14, no. 3, pp. 161-174, 1993.

[4] M. Norrlöf and S. Gunnarsson, "An adaptive iterative learning control algorithm with experiments on an industrial robot," IEEE Transactions on robotics and automation, vol. 18, no. 2, pp. 245-251, 2002.

[5] H. Elci, R. Longman, M. Phan, J. Juang, and R. Ugoletti, "Simple learning control made practical by zero-phase filtering: Applications to robotics," IEEE Transactions on circuits and systems, vol. 49, no. 6 , pp. 753-767, June 2002.

[6] M. Norrlöf, "Iterative learning control, analysis, design and experiments," PhD-thesis, Linköpings universitet, 2000.

[7] F. Miyazaki, S. Kawamura, M. Matsumori, and S. Arimoto, "Learning control scheme for a class of robot systems with elasticity," in Proceedings of 25th Conference on Decision and Control. Athens, Greece: IEEE, December 1986, pp. 74-79.

[8] A. D. Luca and G. Ulivi, "Iterative learning control of robots with elastic joints," in Proceedings of the IEEE International conference on Robotics and Automation. Nice, France: IEEE, May 1992, pp. 1920-1926.

[9] M. Wada, T. Tsukarhara, and K. Tsuda, "Learning control of elastic joint robot and its application to the industrial robot manipulator," in Proceedings of the IEEE International confeence on Robotics and Automation, IEEE. Piscataway, NJ, USA: IEEE, 1993, pp. 417-422.

[10] S. Gunnarsson, M. Norrlöf, E. Rahic, and M. Özbek, "Iterative learning control of a flexible robot arm using accelerometers," in Proceedings of the IEEE International Conference on Control Applications. IEEE, 2004, pp. 1012-1016.

[11] W. Hakvoort, R. Aarts, J. van Dijk, and J. Jonker, "Iterative learning control for improved end-effector accuracy of an industrial robot," in Proceedings of Symposium on Robot Control. IFAC, September 2006.

[12] N. Amann, D. Owens, and E. Rogers, "Iterative learning control for discrete-time systems with exponential rate of convergence," IEE Proc.-Control Theory Appl., vol. 143, no. 2, pp. 217-224, March 1996.

[13] W. Hakvoort, R. Aarts, J. van Dijk, and J. Jonker, "An efficient implementation of optimal ilc for ltv systems," submitted for publication in Automatica, 2007.

[14] L. Ljung, System Identification - Theory For the User, 2nd ed. Upper Saddle River, N.J., USA: PTR Prentice Hall, 1999.

[15] F. Pertin and J. Bonnet-des-Tuves, "Real time robot controller abstraction layer," in Proceedings of the International Symposium on Robotics 2004, 2004

[16] P. Corke, "A robotics toolbox for MATLAB," IEEE Robotics and Automation Magazine, vol. 3, no. 1, pp. 24-32, March 1996.

[17] M. de Graaf, R. Aarts, J. Meijer, and J. Jonker, "Robot-sensor synchronization for real-time seam-tracking in robotic laser welding." in Proceedings of the third International WLT-conference on Lasers in Manufacturing, E. Beyer, F. Dausinger, A. Ostendorf, and A. Otto, Eds. Stuttgart: AT-Fachverlag GmbH, June 2005, pp. 419-424. 